\title{
Inclusion relations for certain families of integral operators associated with conic regions
}

\author{
Shahid Mahmood ${ }^{1 *}$ (D), Imran Khan ${ }^{1}$, Hari Mohan Srivastava ${ }^{2,3}$ and Sarfraz Nawaz Malik
}

"Correspondence:

shahidmahmood757@gmail.com

'Department of Mechanical

Engineering, Sarhad University of

Science \& I. T, Peshawar, Pakistan

Full list of author information is

available at the end of the article

\section{Springer}

\begin{abstract}
In this work, we introduce certain subclasses of analytic functions involving the integral operators that generalize the class of uniformly starlike, convex, and close-to-convex functions with respect to symmetric points. We then establish various inclusion relations for these newly defined classes.

MSC: $30 C 45 ; 30 C 50$

Keywords: Sakaguchi functions; Schwarz function; Subordination; Functions with positive real parts; Analytic functions; Conic domain; Uniformly starlike; Integral operators; Symmetrical points
\end{abstract}

\section{Introduction}

Let $\mathcal{A}$ be the class of functions

$$
f(z)=z+\sum_{n=2}^{\infty} a_{n} z^{n}
$$

analytic in the open unit disc $\mathfrak{A}=\{z \in \mathbb{C}:|z|<1\}$, and let $\mathcal{S}$ be the class of functions in $\mathcal{A}$ that are univalent in $\mathfrak{A}$. Also let $\mathcal{S}^{*}, \mathcal{C}, \mathcal{K}$, and $\mathcal{C}^{*}$ be the subclasses of $\mathcal{A}$ consisting of all functions that are starlike, convex, close-to-convex, and quasiconvex, respectively; for details, see [1].

Let $f$ and $g$ be analytic in $\mathfrak{A}$. We say that $f$ is subordinate to $g$, written as $f(z) \prec g(z)$, if there exists a Schwarz function $w$ that is analytic in $\mathfrak{A}$ with $w(0)=0$ and $|w(z)|<1(z \in \mathfrak{A})$ and such that $f(z)=g(w(z))$. In particular, when $g$ is univalent, then such a subordination is equivalent to $f(0)=g(0)$ and $f(\mathfrak{A}) \subseteq g(\mathfrak{A})$; see [1].

Two points $A$ and $A^{\prime}$ are said to be symmetrical with respect to $M$ if $M$ is the midpoint of the line segment $A A^{\prime}$. Sakaguchi [2] introduced and studied the class $\mathcal{S}_{s}^{*}$ of starlike functions with respect to symmetrical points $z$ and $-z$ belonging to the open unit disc $\mathfrak{A}$. The class $\mathcal{S}_{s}^{*}$ includes the classes of convex and odd starlike functions with respect to the origin. It was shown [2] that a necessary and sufficient condition for $f(z) \in \mathcal{S}_{s}^{*}$ to be univalent and starlike with respect to symmetrical points in $\mathfrak{A}$ is that

$$
\frac{2 z f^{\prime}(z)}{f(z)-f(-z)} \in \mathcal{P}, \quad z \in \mathfrak{A}
$$

(c) The Author(s) 2019. This article is distributed under the terms of the Creative Commons Attribution 4.0 International License (http://creativecommons.org/licenses/by/4.0/), which permits unrestricted use, distribution, and reproduction in any medium, provided you give appropriate credit to the original author(s) and the source, provide a link to the Creative Commons license, and indicate if changes were made. 
Das and Singh [3] defined the classes $\mathcal{C}_{s}$ of convex functions with respect to symmetrical points and showed that a necessary and sufficient condition for $f(z) \in \mathcal{C}_{s}$ is that

$$
\frac{2\left(z f^{\prime}(z)\right)^{\prime}}{(f(z)-f(-z))^{\prime}} \in \mathcal{P}, \quad z \in \mathfrak{A} .
$$

It is also well known [3] that $f(z) \in \mathcal{C}_{s}$ if and only if $z f(z) \in \mathcal{S}_{s}^{*}$.

The classes $k-\mathcal{C} \mathcal{V}$ and $k-\mathcal{S} \mathcal{T}$ with $k \geq 0$ denote the famous classes of $k$-uniformly convex and $k$-starlike functions, respectively, introduced by Kanas and Wisniowska, respectively. For some details see [4-7].

Consider the domain

$$
\Omega_{k}=\left\{u+i v ; u>k \sqrt{(u-1)^{2}+v^{2}}\right\} .
$$

For fixed $k, \Omega_{k}$ represents the conic region bounded successively by the imaginary axis $(k=0)$, the right branch of a hyperbola $(0<k<1)$, a parabola $(k=1)$, and an ellipse $(k>1)$. This domain was studied by Kanas [4-6]. The function $p_{k}$ with $p_{k}(0)=1$ and $p_{k}^{\prime}(0)>0$ plays the role of extremal and is given by

$$
p_{k}(z)= \begin{cases}\frac{1+z}{1-z}, & k=0, \\ 1+\frac{2}{\pi^{2}}\left(\log \frac{1+\sqrt{z}}{1-\sqrt{z}}\right)^{2}, & k=1, \\ 1+\frac{2}{1-k^{2}} \sinh ^{2}\left[\left(\frac{2}{\pi} \arccos k\right) \operatorname{arctanh} \sqrt{z}\right], & 0<k<1, \\ 1+\frac{1}{k^{2}-1} \sin \left[\frac{\pi}{2 R(t)} \int_{0}^{\frac{u(z)}{\sqrt{t}}} \frac{1}{\sqrt{1-x^{2}} \sqrt{1-(t x)^{2}}} d x\right]+\frac{1}{k^{2}-1}, & k>1,\end{cases}
$$

with $u(z)=\frac{z-\sqrt{t}}{1-\sqrt{t z}}, t \in(0,1), z \in E$, and $t$ chosen such that $k=\cosh \left(\frac{\pi R^{\prime}(t)}{4 R(t)}\right)$, where $R(t)$ is Legendre's complete elliptic integral of the first kind, and $R^{\prime}(t)$ is the complementary integral of $R(t)$ (see [5, 6]). Let $\mathcal{P}_{p_{k}}$ denote the class of all functions $p(z)$ that are analytic in $E$ with $p(0)=1$ and $p(z) \prec p_{k}(z)$ for $z \in E$. Clearly, we can see that $\mathcal{P}_{p_{k}} \subset \mathcal{P}$, where $\mathcal{P}$ is the class of functions with positive real parts (see [1]). More precisely,

$$
\mathcal{P}_{p_{k}} \subset \mathcal{P}\left(\frac{k}{1+k}\right) \subset \mathcal{P} \text {. }
$$

For more detail regarding conic domains and related classes, see [4-6, 8-11].

Recently, Noor [12] defined the classes $k-\mathcal{S} \mathcal{T}_{s}, k-\mathcal{U C} \mathcal{V}_{s}$, and $k-\mathcal{U} \mathcal{K}_{s}$ of $k$-uniformly starlike, convex, and close to convex functions with respect to symmetrical points and studied various interesting properties for these classes.

We consider the following one-parameter families of integral operators:

$$
\begin{aligned}
& \mathcal{I}_{\beta}^{\alpha} f(z)=\frac{(\beta+1)^{\alpha}}{\Gamma(\alpha) z^{\beta}} \int_{0}^{z} t^{\beta-1}\left(\log \frac{z}{t}\right)^{\alpha-1} f(t) d t, \\
& \mathfrak{L}_{\beta}^{\alpha} f(z)=\left(\begin{array}{c}
\alpha+\beta \\
\beta
\end{array}\right) \frac{\alpha}{z^{\beta}} \int_{0}^{z} t^{\beta-1}\left(1-\frac{t}{z}\right)^{\alpha-1} f(t) d t,
\end{aligned}
$$

and

$$
\mathfrak{J}_{\beta} f(z)=\frac{\beta+1}{z^{\beta}} \int_{0}^{z} t^{\beta-1} f(t) d t,
$$


where $\alpha \geq 0, \beta>-1$, and $\Gamma$ is the familiar gamma function. We note that $\mathfrak{J}_{\beta}: \mathcal{A} \rightarrow \mathcal{A}$ defined by (1.6) is the generalized Bernardi operator introduced in [13] for $\beta=1,2,3, \ldots$, and for any real number $\beta>-1$, this operator was studied by Owa and Srivastava [14, 15]. For the operators $\mathfrak{L}_{\beta}^{\alpha}$ and $\mathcal{I}_{\beta}^{\alpha}$, we refer to [16, 17]. Also, for $\alpha=1$, we see that

$$
\mathfrak{J}_{\beta} f(z)=\mathfrak{L}_{\beta}^{1} f(z)=\mathcal{I}_{\beta}^{1} f(z) .
$$

We can represent these operators as follows:

$$
\begin{aligned}
\mathcal{I}_{\beta}^{\alpha} f(z) & =z+\sum_{n=2}^{\infty}\left(\frac{\beta+1}{\beta+n}\right)^{\alpha} a_{n} z^{n} \\
& =\left(z+\sum_{n=2}^{\infty}\left(\frac{\beta+1}{\beta+n}\right)^{\alpha} z^{n}\right) * f(z), \\
\mathfrak{L}_{\beta}^{\alpha} f(z) & =z+\sum_{n=2}^{\infty} \frac{\Gamma(\beta+n) \Gamma(\alpha+\beta+1)}{\Gamma(\alpha+\beta+n) \Gamma(\beta+1)} a_{n} z^{n} \\
& =\left(\begin{array}{c}
\alpha+\beta \\
\beta
\end{array}\right) z_{2} F_{1}(1, \beta ; \alpha+\beta ; z) * f(z),
\end{aligned}
$$

and

$$
\mathfrak{J}_{\beta} f(z)=z+\sum_{n=2}^{\infty}\left(\frac{\beta+1}{\beta+n}\right) a_{n} z^{n}
$$

where ${ }_{2} F_{1}$ denotes the Gaussian hypergeometric function, and the symbol $*$ stands for the convolution (Hadamard product).

By (1.7) and (1.8) we can easily derive the identities

$$
z\left(\mathcal{I}_{\beta}^{\alpha} f(z)\right)^{\prime}=(\beta+1) \mathcal{I}_{\beta}^{\alpha-1} f(z)-\beta \mathcal{I}_{\beta}^{\alpha} f(z)
$$

and

$$
z\left(\mathfrak{L}_{\beta}^{\alpha} f(z)\right)^{\prime}=(\alpha+\beta) \mathfrak{L}_{\beta}^{\alpha-1} f(z)-(\alpha+\beta-1) \mathfrak{L}_{\beta}^{\alpha} f(z),
$$

where $\alpha \geq 1$ and $\beta>-1$. From (1.10) we have

$$
\left[\frac{1}{1+\beta} p(z)+\frac{\beta}{1+\beta}\right]=\frac{\mathcal{I}_{\beta}^{\alpha-1} f(z)}{\mathcal{I}_{\beta}^{\alpha} f(z)}
$$

with

$$
p(z)=\frac{z\left(\mathcal{I}_{\beta}^{\alpha} f(z)\right)^{\prime}}{\mathcal{I}_{\beta}^{\alpha} f(z)} .
$$

With the help of these integral operators, we now define the following classes.

Definition 1.1 Let $f(z) \in \mathcal{A}$. Then $f(z) \in k-\mathcal{S} \mathcal{T}_{s}(\alpha, \beta), \alpha \geq 0, \beta>-1$, if $\mathcal{I}_{\beta}^{\alpha} f(z) \in k-\mathcal{S} \mathcal{T}_{s}$ in $\mathfrak{A}$. 
Definition 1.2 Let $f(z) \in \mathcal{A}$. Then $f(z) \in k-\mathcal{S T}_{s}^{*}(\alpha, \beta), \alpha \geq 0, \beta>-1$, if $\mathfrak{L}_{\beta}^{\alpha} f(z) \in k-\mathcal{S} \mathcal{T}_{s}$ in $\mathfrak{A}$.

Definition 1.3 Let $f(z) \in \mathcal{A}$. Then $f(z) \in k-\mathcal{U} \mathcal{K}_{s}(\alpha, \beta), \alpha \geq 0, \beta>-1$, if $\mathcal{I}_{\beta}^{\alpha} f(z) \in k-\mathcal{U} \mathcal{K}_{s}$ in $\mathfrak{A}$.

Definition 1.4 Let $f(z) \in \mathcal{A}$. Then $f(z) \in k-\mathcal{U} \mathcal{K}_{s}^{*}(\alpha, \beta), \alpha \geq 0, \beta>-1$, if $\mathfrak{L}_{\beta}^{\alpha} f(z) \in k-\mathcal{U} \mathcal{K}_{s}$ in $\mathfrak{A}$.

\section{A set of lemmas}

In this section, we give the following lemmas, which will be used in our investigation.

Lemma 2.1 ([4]) Let $k \geq 0$, and let $\beta_{1}, \gamma \in \mathbb{C}$ be such that $\beta_{1} \neq 0$ and $\mathfrak{R e}\left\{\frac{\beta_{1} k}{k+1}+\gamma\right\}>0$. Suppose that $p(z)$ is analytic in $\mathfrak{A}$ with $p(0)=1$ and satisfies

$$
\left(p(z)+\frac{z p^{\prime}(z)}{\beta_{1} p(z)+\gamma}\right) \prec p_{k}(z)
$$

and that $q(z)$ is an analytic function satisfying

$$
q(z)+\frac{z q^{\prime}(z)}{\beta_{1} q(z)+\gamma}=p_{k}(z)
$$

Then $q(z)$ is univalent, $p(z) \prec q(z) \prec p_{k}(z)$, and $q(z)$ is the best dominant of $(2.1)$ given as

$$
q(z)=\left[\beta_{1} \int_{0}^{1}\left(t^{\beta_{1}+\gamma-1} \exp \int_{z}^{t z} \frac{p_{k}(u)-1}{u} d u\right) d t\right]^{-1}-\frac{\gamma}{\beta_{1}}
$$

Lemma 2.2 ([18]) Let $\lambda, \rho \in \mathbb{C}$ be such that $\lambda \neq 0$, and let $\phi(z) \in \mathcal{A}$ be convex and univalent in $\mathbb{U}$ with $\mathfrak{R e}\{\lambda \phi(z)+\rho\}>0(z \in \mathbb{U})$. Also, let $q(z) \in \mathcal{A}$ and $q(z) \prec \phi(z)$. If $p(z)$ is analytic in $\mathbb{U}$ with $p(0)=1$ and satisfies

$$
\left(p(z)+\frac{z p^{\prime}(z)}{\lambda q(z)+\rho}\right) \prec \phi(z)
$$

then $p(z) \prec \phi(z)$.

\section{The main results and their consequences}

Our first main result is stated as the following:

Theorem 3.1 Let $f(z) \in k-\mathcal{S} \mathcal{T}_{s}(\alpha, \beta)$. Then the odd function

$$
\psi(z)=\frac{1}{2}[f(z)-f(-z)] \in k-\mathcal{S} \mathcal{T}(\alpha, \beta) .
$$

Proof Note that

$$
\mathcal{I}_{\beta}^{\alpha} \psi(z)=\frac{1}{2}\left[\mathcal{I}_{\beta}^{\alpha} f(z)-\mathcal{I}_{\beta}^{\alpha} f(-z)\right]
$$


We want to show that $\mathcal{I}_{\beta}^{\alpha} \psi(z) \in k-\mathcal{S T}$. Now, for $f(z) \in k-\mathcal{S} \mathcal{T}_{s}(\alpha, \beta)$, this implies that $\mathcal{I}_{\beta}^{\alpha} f(z) \in k-\mathcal{S} \mathcal{T}_{s}$. Then, for $z \in \mathfrak{A}$,

$$
\begin{aligned}
\frac{z\left(\mathcal{I}_{\beta}^{\alpha} \psi(z)\right)^{\prime}}{\mathcal{I}_{\beta}^{\alpha} \psi(z)} & =\frac{1}{2}\left[\frac{2 z\left(\mathcal{I}_{\beta}^{\alpha} f(z)\right)^{\prime}}{\mathcal{I}_{\beta}^{\alpha} f(z)-\mathcal{I}_{\beta}^{\alpha} f(-z)}+\frac{2(-z)\left(\mathcal{I}_{\beta}^{\alpha} f(-z)\right)^{\prime}}{\mathcal{I}_{\beta}^{\alpha} f(-z)-\mathcal{I}_{\beta}^{\alpha} f(z)}\right] \\
& =\frac{1}{2}\left[h_{1}(z)+h_{2}(z)\right] \\
& =h(z) .
\end{aligned}
$$

and $h_{i}(z) \prec p_{k}(z), i=1,2$. This implies that $h(z) \prec p_{k}(z)$ in $\mathfrak{A}$, and therefore $\mathcal{I}_{\beta}^{\alpha} \psi(z) \in k-$ $\mathcal{S T}$. Consequently, $\psi(z) \in k-\mathcal{S} \mathcal{T}(\alpha, \beta)$ in $\mathfrak{A}$.

Similarly, we can prove that if $f(z) \in k-\mathcal{S T}_{s}^{*}(\alpha, \beta)$, then

$$
\phi(z)=\frac{1}{2}[f(z)-f(-z)] \in k-\mathcal{S T}^{*}(\alpha, \beta) .
$$

Taking $\alpha=0$, we obtain the following result proved by Noor [12].

Corollary 3.2 Let $f(z) \in k-\mathcal{S} \mathcal{T}_{s}$. Then the odd function

$$
\psi(z)=\frac{1}{2}[f(z)-f(-z)] \in k-\mathcal{S} \mathcal{T} .
$$

Note that, for $k=\alpha=0$, the function $\psi(z)=\frac{1}{2}[f(z)-f(-z)]$ is a starlike function in $\mathfrak{A}$; see [2].

Theorem 3.3 Let $\alpha \geq 2$ and $\beta>-1$. Then $k-\mathcal{S T}(\alpha-1, \beta) \subset k-\mathcal{S T}(\alpha, \beta)$.

Proof Let $f(z) \in k-\mathcal{S T}(\alpha-1, \beta)$ and set

$$
p(z)=\frac{z\left(\mathcal{I}_{\beta}^{\alpha} f(z)\right)^{\prime}}{\mathcal{I}_{\beta}^{\alpha} f(z)} .
$$

Note that $p(z)$ is analytic in $\mathfrak{A}$ with $p(0)=1$.

From (3.1) and identity (1.10) we have

$$
\frac{\mathcal{I}_{\beta}^{\alpha-1} f(z)}{\mathcal{I}_{\beta}^{\alpha} f(z)}=(1-\gamma) p(z)+\gamma
$$

with

$$
\gamma=\frac{\beta}{\beta+1} .
$$

Logarithmic differentiation of (3.2) yields

$$
\frac{z\left(\mathcal{I}_{\beta}^{\alpha-1} f(z)\right)^{\prime}}{\mathcal{I}_{\beta}^{\alpha-1} f(z)}=\left\{p(z)+\frac{(1-\gamma) z p^{\prime}(z)}{(1-\gamma) z p(z)+\gamma}\right\},
$$


and thus it follows that

$$
\left(p(z)+\frac{z p^{\prime}(z)}{z p(z)+\beta}\right) \prec p_{k}(z) .
$$

Using Lemma 2.1, we have

$$
p(z) \prec q(z) \prec p_{k}(z)
$$

with

$$
q(z)=\left[\int_{0}^{1}\left(t^{\beta} \exp \int_{z}^{t z} \frac{p_{k}(u)-1}{u} d u\right) d t\right]^{-1}-\beta .
$$

This proves that $f(z) \in k-\mathcal{S T}(\alpha, \beta)$ in $\mathfrak{A}$, and the proof is complete.

Theorem 3.4 Let $\alpha \geq 2$ and $\beta>-1$. Then $k-\mathcal{S T}^{*}(\alpha-1, \beta) \subset k-\mathcal{S T}^{*}(\alpha, \beta)$.

Proof Let

$$
\frac{z\left(\mathfrak{L}_{\beta}^{\alpha} f(z)\right)^{\prime}}{\mathfrak{L}_{\beta}^{\alpha} f(z)}=h(z),
$$

where $h(z)$ is analytic in $\mathfrak{A}$ with $h(0)=1$.

From (3.4) and identity (1.11) we get

$$
\frac{1}{\alpha+\beta} \frac{z\left(\mathfrak{L}_{\beta}^{\alpha} f(z)\right)^{\prime}}{\mathfrak{L}_{\beta}^{\alpha} f(z)}+\left(1-\frac{1}{\alpha+\beta}\right)=\frac{\mathfrak{L}_{\beta}^{\alpha-1} f(z)}{\mathfrak{L}_{\beta}^{\alpha} f(z)} .
$$

Logarithmic differentiation of (3.5), together with (3.4), gives us

$$
\begin{aligned}
\frac{z\left(\mathfrak{L}_{\beta}^{\alpha-1} f(z)\right)^{\prime}}{\mathfrak{L}_{\beta}^{\alpha-1} f(z)} & =h(z)+\frac{\frac{1}{\alpha+\beta} z h^{\prime}(z)}{\frac{1}{\alpha+\beta} h(z)+\frac{\alpha+\beta-1}{\alpha+\beta}} \\
& =h(z)+\frac{z h^{\prime}(z)}{h(z)+\alpha+\beta-1} .
\end{aligned}
$$

Since $f(z) \in k-\mathcal{S T}^{*}(\alpha-1, \beta)$, it follows that

$$
h(z)+\frac{z h^{\prime}(z)}{h(z)+\alpha+\beta-1} \prec p_{k}(z) .
$$

Applying Lemma 2.1, we have

$$
h(z) \prec p_{k}(z) .
$$

This proves our result.

Theorem 3.5 Let $\alpha \geq 2$ and $\beta>-1$. Then $k-\mathcal{S T}_{s}(\alpha-1, \beta) \subset k-\mathcal{S} \mathcal{T}_{s}(\alpha, \beta)$. 
Proof Let $f(z) \in k-\mathcal{S} \mathcal{T}_{s}(\alpha-1, \beta)$. Then, using Theorems 3.1 and 3.3, we have

$$
\psi(z)=\frac{f(z)-f(-z)}{2} \in k-\mathcal{S} \mathcal{T}(\alpha-1, \beta) \subset k-\mathcal{S} \mathcal{T}(\alpha, \beta)
$$

From this it easily follows that $f(z) \in k-\mathcal{S} \mathcal{T}_{s}(\alpha, \beta)$, and this completes the proof.

A similar result for the class $k-\mathcal{S} \mathcal{T}_{s}^{*}(\alpha, \beta)$ can be easily proved.

Theorem 3.6 Let $\alpha \geq 1$ and $\beta>0$. Then $k-\mathcal{U} \mathcal{K}_{s}(\alpha-1, \beta) \subset k-\mathcal{U} \mathcal{K}_{s}(\alpha, \beta)$.

Proof Let $f(z) \in k-\mathcal{U} \mathcal{K}_{s}(\alpha-1, \beta)$. Then there exists $g(z) \in k-\mathcal{S} \mathcal{T}_{s}(\alpha-1, \beta)$ such that

$$
\frac{2 z\left(\mathcal{I}_{\beta}^{\alpha-1} f(z)\right)^{\prime}}{\mathcal{I}_{\beta}^{\alpha-1} g(z)-\mathcal{I}_{\beta}^{\alpha-1} g(-z)}=\frac{z\left(\mathcal{I}_{\beta}^{\alpha-1} f(z)\right)^{\prime}}{\mathcal{I}_{\beta}^{\alpha-1} \psi(z)} \in P
$$

where $\psi(z)=\frac{\mathcal{I}_{\beta}^{\alpha-1} g(z)-\mathcal{I}_{\beta}^{\alpha-1} g(-z)}{2} \in k-\mathcal{S} \mathcal{T}(\alpha-1, \beta) \subset k-\mathcal{S T}(\alpha, \beta)$ in $\mathfrak{A}$.

Let us set

$$
\frac{z\left(\mathcal{I}_{\beta}^{\alpha} f(z)\right)^{\prime}}{\mathcal{I}_{\beta}^{\alpha} \psi(z)}=p(z)
$$

where $p(z)$ is analytic in $\mathfrak{A}$ with $p(0)=1$. Then by (3.6) and identity (1.10) we get

$$
\frac{\mathcal{I}_{\beta}^{\alpha-1} \psi(z)}{\mathcal{I}_{\beta}^{\alpha} \psi(z)}=(1-\gamma) p_{0}(z)+\gamma
$$

where $p_{0}(z)=\frac{z\left(\mathcal{I}_{\beta}^{\alpha} \psi(z)\right)^{\prime}}{\mathcal{I}_{\beta}^{\alpha} \psi(z)}$, and $\gamma$ is given by (3.3). Now by simple computations we obtain

$$
\begin{aligned}
\frac{z\left(\mathcal{I}_{\beta}^{\alpha-1} f(z)\right)^{\prime}}{z \mathcal{I}_{\beta}^{\alpha-1} \psi(z)} & =\frac{z\left(\mathcal{I}_{\beta}^{\alpha-1} f(z)\right)^{\prime}}{\mathcal{I}_{\beta}^{\alpha} \psi(z)\left[(1-\gamma) p_{0}(z)+\gamma\right]} \\
& =\frac{z\left[\left(z\left(\mathcal{I}_{\beta}^{\alpha} f(z)\right)^{\prime}\right)^{\prime}\right]+\beta z\left(\mathcal{I}_{\beta}^{\alpha} f(z)\right)^{\prime}}{(\beta+1) \mathcal{I}_{\beta}^{\alpha} \psi(z)\left[(1-\gamma) p_{0}(z)+\gamma\right]} \\
& =\frac{\beta p(z)+p(z) p_{0}(z)+z p^{\prime}(z)}{(\beta+1)\left[\left(1-\frac{\beta}{1+\beta}\right) p_{0}(z)+\frac{\beta}{1+\beta}\right]} \\
& =\frac{\beta p(z)+p(z) p_{0}(z)+z p^{\prime}(z)}{p_{0}(z)+\beta} \\
& =p(z)+\frac{z p^{\prime}(z)}{p_{0}(z)+\beta} .
\end{aligned}
$$

Since $f(z) \in k-\mathcal{U} \mathcal{K}_{s}(\alpha-1, \beta)$, it follows that

$$
p(z)+\frac{z p^{\prime}(z)}{p_{0}(z)+\beta} \in \mathcal{P} \quad \text { in } \mathfrak{A} .
$$

Applying Lemma.2.2, we have $p(z) \in \mathcal{P}$ in $\mathfrak{A}$. This proves $f(z) \in k-\mathcal{U} \mathcal{K}_{s}(\alpha, \beta)$ in $\mathfrak{A}$.

By a similar argument we can easily prove the following inclusion result. 
Theorem 3.7 Let $\alpha \geq 1$ and $\beta>0$. Then $k-\mathcal{U} \mathcal{K}^{*}(\alpha-1, \beta) \subset k-\mathcal{U} \mathcal{K}^{*}(\alpha, \beta)$.

Theorem 3.8 Let $f(z) \in k-\mathcal{S} \mathcal{T}_{s}(\alpha, \beta)$ in $\mathfrak{A}$. Then

$$
\mathfrak{R e}\left\{\frac{z\left(\mathcal{I}_{\beta}^{\alpha-1} f(z)\right)^{\prime}}{\mathcal{I}_{\beta}^{\alpha-1} \varphi(z)}\right\}>0
$$

for $|z|<R\left(\beta, \gamma_{0}\right)$, where

$$
R\left(\beta, \gamma_{0}\right)=\frac{(1+\beta)}{\left(2-\gamma_{0}\right)+\sqrt{\left(2-\gamma_{0}\right)^{2}+(1+\beta)\left(\beta+2 \gamma_{0}-1\right)}}
$$

with

$$
\gamma_{0}=\frac{k}{k+1}
$$

Proof Let $f(z) \in k-\mathcal{S} \mathcal{T}_{s}(\alpha, \beta)$. Then

$$
\varphi(z)=\frac{f(z)-f(-z)}{2} \in k-\mathcal{S} \mathcal{T}(\alpha, \beta)
$$

and hence

$$
\frac{z\left(\mathcal{I}_{\beta}^{\alpha} f(z)\right)^{\prime}}{\mathcal{I}_{\beta}^{\alpha} \varphi(z)} \in \mathcal{P}\left(p_{k}\right) \subset \mathcal{P}\left(\gamma_{0}\right)
$$

where $\gamma_{0}$ is given by (3.7). Let

$$
\begin{aligned}
\frac{z\left(\mathcal{I}_{\beta}^{\alpha} f(z)\right)^{\prime}}{\mathcal{I}_{\beta}^{\alpha} \varphi(z)} & =h(z), \quad h(z) \in \mathcal{P}\left(\gamma_{0}\right), \\
& =\left(1-\gamma_{0}\right) h_{0}(z)+\gamma_{0}, \quad h_{0}(z) \in \mathcal{P} .
\end{aligned}
$$

Then, proceeding as in Theorem 3.5, we have

$$
\frac{z\left(\mathcal{I}_{\beta}^{\alpha-1} f(z)\right)^{\prime}}{\mathcal{I}_{\beta}^{\alpha-1} \varphi(z)}=h(z)+\frac{z h^{\prime}(z)}{p(z)+\beta}
$$

where $p(z)=\frac{z\left(\mathcal{I}_{\beta}^{\alpha} \varphi(z)\right)^{\prime}}{\mathcal{I}_{\beta}^{\alpha} \varphi(z)} \in \mathcal{P}(\gamma)$. Using (3.8) and $p(z)=\left(1-\gamma_{0}\right) p_{0}(z)+\gamma_{0}$ in (3.9), we have

$$
\frac{z\left(\mathcal{I}_{\beta}^{\alpha-1} f(z)\right)^{\prime}}{\mathcal{I}_{\beta}^{\alpha-1} \varphi(z)}=\left(1-\gamma_{0}\right) h_{0}(z)+\gamma_{0}+\frac{\left(1-\gamma_{0}\right) z h_{0}^{\prime}(z)}{\left(1-\gamma_{0}\right) p_{0}(z)+\gamma_{0}+\beta}
$$

with $h_{0}(z) \in \mathcal{P}, p_{0}(z) \in \mathcal{P}$, that is,

$$
\frac{1}{1-\gamma_{0}}\left[\frac{z\left(\mathcal{I}_{\beta}^{\alpha-1} f(z)\right)^{\prime}}{\mathcal{I}_{\beta}^{\alpha-1} \varphi(z)}-\gamma_{0}\right]=h_{0}(z)+\frac{z h_{0}^{\prime}(z)}{\left(1-\gamma_{0}\right) p_{0}(z)+\gamma_{0}+\beta}
$$


Using the distortion result for the class $\mathcal{P}$, we obtain

$$
\begin{aligned}
\mathfrak{R e} & {\left[\frac{1}{1-\gamma_{0}}\left\{\frac{z\left(\mathcal{I}_{\beta}^{\alpha-1} f(z)\right)^{\prime}}{\mathcal{I}_{\beta}^{\alpha-1} \varphi(z)}-\gamma_{0}\right\}\right] } \\
& \geq \mathfrak{R e} h_{0}(z)\left\{1-\frac{\frac{2 r}{1-r^{2}}}{\left(1-\gamma_{0}\right) \frac{1-r}{1+r}+\left(\gamma_{0}+\beta\right)}\right\} \\
& =\mathfrak{R e} h_{0}(z)\left\{1-\frac{2 r}{\left(1-\gamma_{0}\right)(1+r)^{2}+\left(1-r^{2}\right)\left(\gamma_{0}+\beta\right)}\right\} .
\end{aligned}
$$

Right-hand side of (3.10) is greater than or equal to zero for $|z|<R\left(\beta, \gamma_{0}\right)$, where $R\left(\beta, \gamma_{0}\right)$ is the least positive root of the equation

$$
T(r):=\left(1-\beta-2 \gamma_{0}\right) r^{2}-2\left(2-\gamma_{0}\right) r+(1+\beta)=0,
$$

that is,

$$
\begin{aligned}
R\left(\beta, \gamma_{0}\right) & =\frac{2\left(2-\gamma_{0}\right)-\sqrt{4\left(2-\gamma_{0}\right)^{2}+4(1+\beta)\left(\beta+2 \gamma_{0}-1\right)}}{2\left(1-\beta-2 \gamma_{0}\right)} \\
& =\frac{(1+\beta)}{\left(2-\gamma_{0}\right)+\sqrt{\left(2-\gamma_{0}\right)^{2}+(1+\beta)\left(\beta+2 \gamma_{0}-1\right)}} .
\end{aligned}
$$

The proof is completed.

\section{Particular Cases}

(i) For $\beta=0$ and $\gamma_{0}=\frac{k}{k+1}=0$ (i.e., $k=0$ ), we have $f(z) \in \mathcal{S}_{s}^{*}(\alpha, 0)\left(\psi \in \mathcal{S}^{*}(\alpha, 0)\right)$ and

$$
R(0,0)=\frac{1}{2+\sqrt{3}}
$$

(ii) For $k=1$ and $\beta=0$,

$$
R\left(0, \frac{1}{2}\right)=\frac{1}{3}
$$

(iii) For $k=1$ and $\beta=1$,

$$
R\left(1, \frac{1}{2}\right)=\frac{4}{4+\sqrt{17}}
$$

Theorem 3.9 Let $\mathfrak{L}_{\beta}^{\alpha} f(z) \in k-\mathcal{S} \mathcal{T}$. Then

$$
\mathfrak{L}_{\beta}^{\alpha-1} f(z) \in \mathcal{S}^{*}\left(\gamma_{0}\right), \quad \gamma_{0}=\frac{k}{k+1}
$$

for $|z|<R_{1}$, where

$$
R_{1}\left(\alpha, \beta, \gamma_{0}\right)=\frac{\alpha+\beta}{2-\gamma_{0}+\sqrt{\left(2-\gamma_{0}\right)^{2}+(\alpha+\beta)\left(2 \gamma_{0}+\alpha+\beta-2\right)}} .
$$


Proof Since $\mathfrak{L}_{\beta}^{\alpha} f(z) \in k-\mathcal{S T}$, we have

$$
\frac{z\left(\mathfrak{L}_{\beta}^{\alpha} f(z)\right)^{\prime}}{\mathfrak{L}_{\beta}^{\alpha} f(z)}=h(z), \quad h(z) \prec p_{k}(z)
$$

in $\mathfrak{A}$. With a similar argument as in Theorem 3.5, we have

$$
\frac{z\left(\mathfrak{L}_{\beta}^{\alpha-1} f(z)\right)^{\prime}}{\mathfrak{L}_{\beta}^{\alpha-1} f(z)}=h(z)+\frac{z h^{\prime}(z)}{h(z)+\alpha+\beta-1},
$$

that is,

$$
\begin{aligned}
\mathfrak{R e} & {\left[\frac{1}{1-\gamma_{0}}\left\{\frac{z\left(\mathfrak{L}_{\beta}^{\alpha-1} f(z)\right)^{\prime}}{\mathfrak{L}_{\beta}^{\alpha-1} f(z)}-\gamma_{0}\right\}\right] } \\
& =\mathfrak{R e}\left[h_{0}(z)+\frac{z h_{0}^{\prime}(z)}{\left(1-\gamma_{0}\right) h_{0}(z)+\left(\gamma_{0}+\alpha+\beta-1\right)}\right] \\
& \geq \mathfrak{R e} h_{0}(z)\left[1-\frac{\frac{2 r}{1-r^{2}}}{\left(1-\gamma_{0}\right) \frac{1-r}{1+r}+\left(\gamma_{0}+\alpha+\beta-1\right)}\right],
\end{aligned}
$$

where

$$
h(z)=\left(1-\gamma_{0}\right) h_{0}(z)+\gamma_{0}, \quad h_{0} \in \mathcal{P}, \gamma_{0}=\frac{k}{k+1} .
$$

The right-hand side of (3.11) is greater than or equal to zero for $|z|<R_{1}$, where $R_{1}$ is the least positive root of the equation

$$
T(r):=\left(2-2 \gamma_{0}-\alpha-\beta\right) r^{2}-2\left(2-\gamma_{0}\right) r+\alpha+\beta=0
$$

that is,

$$
\begin{aligned}
R_{1}\left(\alpha, \beta, \gamma_{0}\right) & =\frac{2-\gamma_{0}-\sqrt{\left(2-\gamma_{0}\right)^{2}+(\alpha+\beta)\left(2 \gamma_{0}+\alpha+\beta-2\right)}}{2\left(2-\alpha-\beta-2 \gamma_{0}\right)} \\
& =\frac{\alpha+\beta}{2-\gamma_{0}+\sqrt{\left(2-\gamma_{0}\right)^{2}+(\alpha+\beta)\left(2 \gamma_{0}+\alpha+\beta-2\right)}} .
\end{aligned}
$$

This completes the proof.

\section{Conclusion}

In this paper, we have defined some new classes of analytic functions involving integral operators. We have shown that these classes generalize the well-known classes, and already existing results can be obtained as a particular cases of our results. Inclusion relations of these classes are also a significant part of our work. We believe that the work presented in this paper will give researchers a new direction and will motivate them to explore more interesting facts on similar lines.

\section{Acknowledgements}

The authors would like to thank the reviewers of this paper for his/her valuable comments on the earlier version of the paper. They would also like to acknowledge Prof. Dr. Salim ur Rehman, V.C. Sarhad University of Science \& I. T, for providing excellent research and academic environment. 
Funding

Sarhad University of Science \& I. T Peshawar.

\section{Availability of data and materials}

Not applicable.

\section{Competing interests}

The authors declare that they have no competing interests.

Authors' contributions

All authors jointly worked on the results, and they read and approved the final manuscript.

\section{Author details}

${ }^{1}$ Department of Mechanical Engineering, Sarhad University of Science \& I. T, Peshawar, Pakistan. ${ }^{2}$ Department of Mathematics and Statistics, University of Victoria, Victoria, Canada. ${ }^{3}$ Department of Medical Research, China Medical University Hospital, China Medical University, Taichung, Taiwan, Republic of China. ${ }^{4}$ Department of Mathematics, COMSATS University Islamabad, Wah Campus, Pakistan.

\section{Publisher's Note}

Springer Nature remains neutral with regard to jurisdictional claims in published maps and institutional affiliations.

Received: 14 November 2018 Accepted: 27 February 2019 Published online: 07 March 2019

\section{References}

1. Goodman, A.W.: Univalent Functions, Vol. I, II. Polygonal Publishing House, Washington (1983)

2. Sakaguchi, K.: On a certain univalent mapping. J. Math. Soc. Jpn. 11, $72-73$ (1959)

3. Das, R.N., Singh, P.: On subclasses of Schlicht mappings. Indian J. Pure Appl. Math. 8, 864-872 (1977)

4. Kanas, S.: Techniques of the differential subordination for domains bounded by conic sections. Int. J. Math. Math. Sci. 38, 2389-2400 (2003)

5. Kanas, S., Wisniowska, A.: Conic regions and k-uniform convexity. J. Comput. Appl. Math. 105, 327-336 (1999)

6. Kanas, S., Wisniowska, A.: Conic domains and starlike functions. Rev. Roum. Math. Pures Appl. 45, 647-657 (2000)

7. Kanas, S., Srivastava, H.M.: Linear operators associated with k-uniformly convex functions. Integral Transforms Spec. Funct. 9, 121-132(2000)

8. Arif, M., Mahmood, S., Sokoł, J., Dziok, J.: New subclass of analytic functions in conical domain associated with a linear operator. Acta Math. Sci. 36(3), 1-13 (2016)

9. Arif, M., Umar, S., Mahmood, S., Sokoł, J.: New reciprocal class of analytic functions associated with linear operator. Iran. J. Sci. Technol., Trans. A, Sci. 42(2), 881-886 (2018). https://doi.org/10.1007/s40995-016-0059-y

10. Mahmood, S., Sokoł, J.: New subclass of analytic functions in conical domain associated with Ruscheweyh Q-differential operator. Results Math. 71(3-4), 1345-1357 (2017). https://doi.org/10.1007/S00025-016-0592-1

11. Ul-Haq, W., Mahmood, S.: Certain properties of a class of close-to-convex functions related to conic domains. Abstr. Appl. Anal. 2013, Article ID 847287, 1-6 (2013)

12. Noor, K.I.: On uniformly univalent functions with respect to symmetrical points. J. Inequal. Appl. 2014, 254, 1-14 (2014)

13. Bernardi, S.D.: Convex and starlike univalent functions. Trans. Am. Math. Soc. 135, 429-446 (1969)

14. Owa, S., Srivastava, H.M.: Some applications of the generalized Libera operator. Proc. Jpn. Acad., Ser. A, Math. Sci. 62, 125-128 (1986)

15. Srivastava, H.M., Owa, S.: A certain one-parameter additive family of operators defined on analytic functions. J. Math. Anal. Appl. 118, 80-87 (1986)

16. Jung, I.B., Kim, Y.C., Srivastava, H.M.: The Hardy space of analytic functions associated with certain one parameter families of integral operator. J. Math. Anal. Appl. 176, 138-147 (1993)

17. Owa, S.: Properties of certain integral operators. Georgian Math. J. 2, 535-545 (1995)

18. Yuan, S.M., Liu, Z.M.: Some properties of $\alpha$-convex and $\alpha$-quasiconvex functions with respect to $n$-symmetric points. Appl. Math. Comput. 188, 1142-1150 (2007)

\section{Submit your manuscript to a SpringerOpen ${ }^{\circ}$ journal and benefit from:}

- Convenient online submission

- Rigorous peer review

- Open access: articles freely available online

- High visibility within the field

Retaining the copyright to your article

Submit your next manuscript at $>$ springeropen.com 